\title{
REINVENCIÓN DE LA MUJER EMPRENDEDORA: UNA REFLEXIÓN DESDE SU AUTONOMÍA ECONÓ- MICA EN BOLIVIA
}

\author{
REINVENTING THE ENTREPRENEURIAL WOMAN: A REFLECTION FROM HER ECONOMIC AU-
}

\section{TONOMY IN BOLIVIA}

MSC. Cristian Calderón Collazos

https://doi.org/10.38147/invneg.v14i23.132

\section{calderon.cristian@usfx.bo}

Docente-Investigador de la Facultad de Ciencias Económicas y Empresariales de la Universi-

dad San Francisco Xavier de Chuquisaca, Bolivia

Recibido: 04 de marzo de 2021

Aprobado: 10 de abril 2021

\begin{abstract}
Resumen
La coyuntura actual trasciende las características de la economía tildada como normal hasta antes del shock COVID-19, pone en descubierto la fragilidad socioeconómica de las personas, afectadas por variables macroeconómicas, ante estos eventos se habla de reinvención empresarial, de una nueva adaptación, sin considerar las estructuras sociales y las características que han limitado el desarrollo de la mujer, si bien se ha intentado que la mujer logre su autonomía con ciertas alternativas entre ellas, el de llevar adelante un emprendimiento, es necesario analizar las condiciones y características con las que se desenvuelven y llevan adelante un negocio, muchos de supervivencia, siendo estas las bases de este proceso investigativo-reflexivo. "Se habla de reinvención pero no de un cambio social".
\end{abstract}

Palabras Clave: Autonomía económica, patriarcado, género.

\begin{abstract}
The current situation transcends the characteristics of the economy classified as normal until before the COVID-19 shock, it reveals the socioeconomic fragility of people, affected by macroeconomic variables, before these events there is talk of business reinvention, a new adaptation, without Consider the social structures and characteristics that have limited the development of women, although it has been tried that women achieve their autonomy with certain alternatives among them, that of carrying out an enterprise, it is necessary to analyze the conditions and characteristics with which they develop and carry out a business, many for survival, these being the bases of this investigative-reflective process. "There is talk of reinvention but not of social change."
\end{abstract}

Key words: Economic autonomy, patriarchy, gender. 


\section{Investigación \& Negocios}

1. El emprendedurismo más allá de una mirada teórica.

En esos instantes de pensamiento fugaz, empírico, bajo una visión limitada asociamos el emprendedurismo hacia una empresa en desarrollo, nos creamos un perfil de un emprendedor dispuesto a asumir el riesgo, sin duda, de contar con capacidades en función a la gestión de su empresa, en ese instante estamos cerca de la definición de Richard Cantillon, aquel que definió que el emprendedurismo implicaba el riesgo económico de una nueva empresa, a él, se le atribuye como el dador de las primeras definiciones del emprendedurismo. Pero, es inevitable no considerar aquellas apreciaciones de Joseph Schumpeter que habla del emprendedor, bajo una mirada admirativa al considerar que es un innovador, aquel que rompe la forma tradicional de hacer las cosas (Carrasco, 2008); el emprendedor un trabajador superior; según Jean Baptiste Say con capacidades en la administración, aquel que optimiza los recursos y vence obstáculos (Osorio, 2010); según Baumol Willan, aquel que es fuente de inspiración, genera ideas y las pone en marcha (Herrera, 2013); o aquel que tiene habilidades que no son comunes al menos así lo señalaba John Sturart Mill.

Y si perdemos ahora esa mirada limitada acerca del emprendedurismo y de aquel emprendedor que a priori llegamos a fijarnos bajo perfiles característicos que nos brinda la teoría clásica, podremos observar entonces de una manera crítica que la conceptualización clásica se perdió en la esencia de una realidad latente en los sistemas de mercado de trabajo, donde el perfil del emprendedor rompe toda una estructura por la singularidad y por la esencia en el que se marcan las distintas formas y características de poder emprender, entonces; al considerar lo mencionado; que emprender no solo es llegar a constituir u negocio si no afrontar y emprender nuevos caminos o nuevos proyectos constantemente sin la necesidad de crear una empresa, un emprendedor también puede ser una persona autónoma que trabaje por cuenta propia de manera independiente (Ferran, P., GuiIlem, P., Albert t., 2015).

\section{En un contexto económico de-}

primido se considera a los emprendedores, abordando nuevos proyectos, son la solución a los problemas que tienen muchas empresas para adaptarse a nuevos tiempos, la solución para acabar con los altos índices de paro: para que los desempleados tengan el aliciente de convertirse en sus propios empleadores, en emprendedores, y que además acaben creando más empleo (Remolins, 2015: 17)

Entonces, con una estructura teórica miope hace que se pierda una mirada y una percepción acerca de la realidad, porque un emprendedor no siempre está dotado de habilidades, de conocimientos no comunes o con capacidades administrativas o gerenciales, sería predecible porque Remolins termina mencionando que para ser emprendedor no hay que ser joven, no hay que ser rico, no hay que haber heredado una empresa. Es verdad que se puede ser emprendedor siendo joven, siendo rico, o partiendo de una empresa familiar, también se puede emprender a una edad madura, empezando desde cero, incluso después de arruinarse

\section{Género, roles, patriarcado.}

Pensar en un estereotipo del emprendedor debe tecnificar no solo lo mencionado por la teoría, el trasfondo radica en establecer conceptos integradores que sean capaces de asimilar la desigualdad social que hablaba Poulain de la Barre citado por Cobo (1995), en la relación hombre y mujer, una desigualdad social y política traducida en la inferioridad de la naturaleza femenina, aquella en la que sus condiciones en el mercado de trabajo se ven supeditados bajo una orden sociocultural (Lagarde, 1996); filosófico, político, religioso subordinadas; (Ramos, 1995), donde las relaciones de poder van a favor de los hombres desde aspectos psicológicos y físicos hasta construir los principios de una sociedad donde radica la desigualdad de género; (Hernández, 2008), aunque la Organización Mundial de la Salud (OMS) menciona que las diferencias entre hombres y mujeres favorecen sistemáticamente a uno de los dos grupos, podemos dar cuenta que las funciones, comportamientos, actividades ya atributos de la 
sociedad tuvo un mejor panorama para los hombres, estar indiferentes a las estadísticas que se muestran estaríamos nuevamente con la miopía social del que hablábamos.

No hablar de desigualdades de género sería como un despropósito de los movimientos femeninos, aquellas que en las décadas 60 y 70, crearon una esencializiación fundamentalmente en Inglaterra y Estados Unidos donde el término "feminismo" estaba basada en la denuncia de la situación de desigualdad y los esfuerzos por propiciar cambios (Espinar, s/F). Sin duda la desigualdad es manifestada mediante la segregación entre hombres y mujeres, (Anker, 1997), donde el mercado de trabajo se considera como una de los sectores donde las diferencias se hacen palpables.

Estas desigualdades de tiempos inexorables no calculadas pero si tipificadas y estudiadas redunda en pensar que los roles asignados a la mujer soslayan el buen actuar del ser humano, más bien, están supeditadas a la organización social en el que la autoridad la ejerce el varón jefe de familia, dueño del patrimonio, del que formaban parte los hijos, la esposa, los esclavos y los empleados, (Fontenla, 2008); creando interdependencia y solidaridad entre ellos, (Hartmann, 1981), y es atribuible considerar que crean una dependencia de la mujer hacia el hombre, no solo en la representación política, plausible considerad la dependencia económica; entonces, en el patriarcado el paradigma es el hombre donde el uso del dominio se basa en ellos mismos enajenando a la mujer manteniendo a las mujeres dependientes de quienes las dominan (Lagarde, 1996).

Creer que los espacios de oportunidades desde la formación en educación, la partición en los espacios de poder y el mercado de trabajo, generaron las mismas condiciones entre hombres y mujeres estuviéramos en una supina descontextualización, la sociedad patriarcal ha construido roles alrededor de la mujer como amas de casa, encargadas de cuidar niños, ancianos, enfermos y discapaci- tados haciendo que exista diferencias en los salarios, acceso a la educación, la salud y la participación política. Estas relaciones patriarcales basadas en una identidad masculina se reproducen en nuestra vida diaria siendo también las mujeres agentes reproductoras de esta sociedad, sin duda esto produjo que las ocupaciones laborales de las mujeres sean dejadas fuera del concepto mismo de trabajo, produciendo concepciones en donde los hombres trabajan y las mujeres son amas de casa, considerando a la mujer una carga para los hombres, y en el mercado laboral se constituyen solo como complemento al verdadero trabajo, que es el que desempeñan los hombres. Por ejemplo el pensamiento retrograda de los empleadores al considerar que asumen un riesgo si contratan mujeres con hijos y si están con pareja (les parece un problema en que ellas lleguen a concebir), mientras que los hombres con familia son una certeza para el empleador (Correa, 2014)"event-place":"Venezuela","ISBN":"978-980-399-053-4","au-

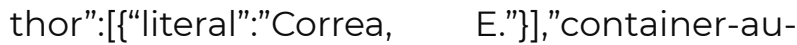
thor":[\{"literal":"Fundación Centro de Estudios Latinoamericanos Rómulo Gallegos"\}],"issued":\{"date-parts":[["2014"]]\}\}\}],"schema":"https:// github.com/citation-style-language/schema/ raw/master/csl-citation.json"\} .

Las condiciones no son las mismas, el hecho en que la sociedad atribuya un peso adicional a la mujer en labores que en muchos países como Bolivia no son consideras ni contabilizadas en su producción nacional hace que vivamos en un modelo que nos parece "normal", y la revalorización está en la medida en formar un modelo económico donde la reivindicación de la mujer esté en el ánimo de la valorización desde las estructuras familiares, desde los niveles iniciales en educación, la inclusión en las decisiones políticas, sociales, económicas incluso religiosas, la formulación de pantallas como espectros a la resolución de estos problemas traducidos en políticas que quieran dirimir la violencia, el racismo, el acoso y la indiferente participación en el mercado, quedarán en una visión sin estrategia, en políticas sin bases, y en una estructura patriar- 
cal sin cambio; el ataque a este sujeto además ha sido ejercido por la misma mujer, aunque suene contradictorio, los modelos de vida impuestos en la mayoría de las ocasiones ha estimulado el rechazo al mismo sexo, a su solidaridad y apoyo induciendo a una competencia exacerbada manifestada en la envidia ante el triunfo de otras, a los estándares de belleza, de formas de vestir o incluso de ser, existen mujeres que relegan a su mismo género a trabajos supeditados, relacionados con la inferioridad y trabajos netamente del hogar.

\section{Entendamos la Autonomía Econó-} mica de la mujer.

Hablar de equidad de género se constituye en una medida que evalúa el logro en el proceso de empoderamiento y superación de la exclusión social, conociendo sus capacidades en decidir de manera autónoma su participación en el mercado; la autonomía económica de las mujeres y la reducción de la pobreza se convierten en los requisitos más importantes para lograr la equidad de género (Heller, 2010), incluyendo una dimensión solidaria y de reconocimiento recíproco, entendiendo que la autonomía económica se entiende como la capacidad de las personas de generar sus propios ingresos que satisfagan sus necesidades (Bravo, 2012); a esto atribuimos que aquel que no genera su propio dinero es pobre, y se hace económicamente dependiente.

Para una mujer tener ingresos se constituye en una base pero, no significa necesariamente ejercer una plena autonomía económica así lo menciona la Comisión para América Latina y el Caribe (CEPAL); los ingresos de las mujeres pueden ser tan bajos que no les permiten superar el umbral de la pobreza, ya sea individual o de sus hogares, importante entonces señalar:

La autonomía económica es un pilar fundamental de la autonomía de las mujeres y por definición, requiere que estas perciban ingresos que les permi- tan superar la pobreza y disponer de su tiempo libremente para capacitarse, acceder al mercado laboral, desarrollarse profesional y personalmente, participar de manera activa de la vida social y política y dedicarse a sus seres queridos sin que ello se transforme en una barrera para el logro de sus propias aspiraciones" (CEPAL, 2016:8).

El documento Las Mujeres escribiendo nuestra Autonomía, impulsado y gestionado por la Organización de Naciones Unidas (ONU) y el Ministerio de Autonomías de Bolivia en 2015, expresa que la autonomía económica es la generación de ingresos sea en un mercado de trabajo e incluso dentro de sus familias, aunque este última consideración se lleva acabo de forma invisibilizada, además el documento expresa que, mediante la autonomía económica se exige la igualdad de trato y remuneración laboral, con un enfoque de políticas de inserción laboral y de fomento a la producción desde las mujeres, considerando incluso que el trabajo del hogar produce riqueza y que esa riqueza debe ser devuelta a ellas; ante esta situación, ahondamos nuevamente en esta discrepancia con la realidad, considerar que el trabajo del hogar debe contabilizarse debe primeramente contar con las bases reglamentarias en cuanto a la cuantificación, mientras tanto, esbozar con la teoría respecto al tema sería improductivo.

La forma y manera de reflexionar el emprendedurismo de estereotipar una figura de un ser de cualidades, habilidades, virtudes y conocimientos quedan enajenados por una realidad perceptible donde la participación de un emprendedor no solo se traduce en la gestión de una empresa, sino que, la lucha por encontrar mejores condiciones, mayores ingresos o simplemente el hecho de sobrevivir hace que las alternativas en la generación de ingresos sean plausibles en la visualización de alternativas que generen movimiento económico.

La mujer desde su posición, aquella que pueda encajar en las definiciones clásicas del emprendedurismo, o aquellas que bajo sus singularidades están en busca de generar in- 
gresos, sea por la necesidad, oportunidad o por una obligación moral que puede ser subjetiva pero latente en las características de cada emprendedora (Calderón, 2017). En la búsqueda que tiene la mujer en la de incrementar su economía o mejorarla, surge las características propias, antes mencionadas, en la que se define el éxito o la precariedad de sus emprendimientos, donde un emprendimiento de subsistencia genera ingresos por debajo del mínimo nacional en Bolivia, en un contexto macro donde se menciona que la autonomía económica es indispensable para el logro de la equidad de género y se encuentran estrechamente relacionados con sus posibilidades de participar en un mercado de trabajo. (Heller, 2010).

\section{Bolivia, contextualizando a la mujer emprendedora.}

a) Participación en la Educación.

La educación, es el factor más importante para el desarrollo de la autonomía económica, que no se tenga duda, en ella se construyen los conocimientos y habilidades de una persona, prescindible tocar este tema considerando que según la Encuesta Mundial de Valores en Bolivia (2017)', la población boliviana considera en un 37\% que la educación universitaria es más importante para los hombres

\section{Gráfico 1}

\section{Especialización educativa en Bolivia}

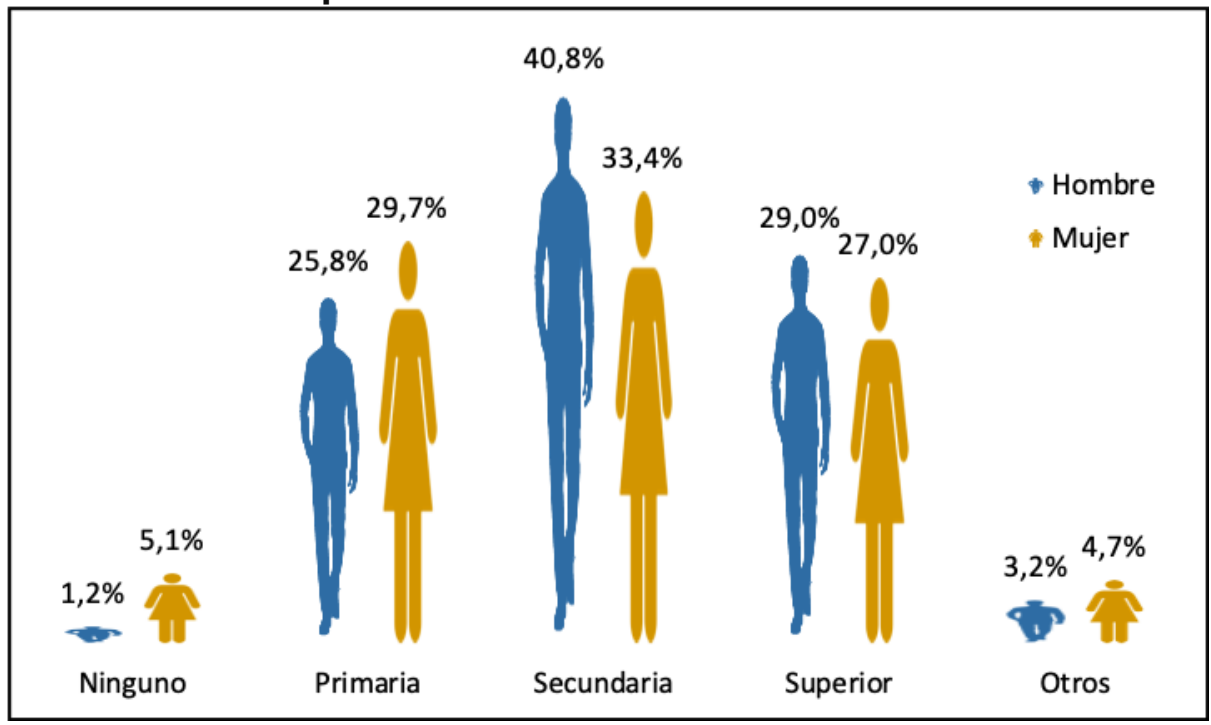

Fuente: Metadatos INE-ECE-3T2019/Elaboración: propia

Es importante reconocer que en los últimos 14 años, a través de planes y programas y fomentos la educación se tornó más equitativa, prueba clara según gráfico 7 es que, tanto hombres y mujeres en cuestión de educación superior ocupan similares porcentajes, bien, veamos esta situación de forma desagregada porque a primera vista parecen datos alentadores, aunque no dejan de serlos, hay más mujeres que terminaron secundaria $(33,4 \%)$ que mujeres con licenciatura (15\%), Hay más mujeres que no saben leer ni escribir $(5,1 \%)$ que mujeres con un posgrado $(2,4 \%)$, cabe notar que las mujeres entre los 40 y 80 años comprenden el grupo de analfabetas a nivel nacional, mientras que el grupo que concentra a los hombres con estas características comprende en 1 La Encuesta Mundial de Valores (EMV) -World Values Survey (WVS), es producto de un convenio interinstitucional suscrito entre la Vicepresidencia del Estado Plurinacional de Bolivia y el Fondo de las Naciones Unidas para la Infancia (UNICEF), el Fondo de Población de las Naciones Unidas (UNFPA), Oxfam y la Comunidad de Estudios Sociales y Acción Pública. 


\section{Investigación \&egocios}

su mayoría entre 60 y 74 años; hay más hombres en universidades privadas $(3,2)$ frente a $(2,6 \%)$ de las mujeres; hay más mujeres en escuelas de formación técnica $(7,8 \%)$ hombres con (7,4\%); la Encuesta nacional de Discriminación y Exclusión Social $(2014)^{2}$, mencionaba que la mayoría de las mujeres en Bolivia solo habían culminado la secundaria con $46 \%$, primaria $23,80 \%$, muy pocas con educación universitaria y superior $12,80 \%$ y educación no universitaria 6,9\%; como se observa, independientemente de la fase técnica preliminar de las bases de datos, la educación para la mujer fue mejorando aunque en términos generales presenta un similar comportamiento, sin duda, la mujer cuenta con menos especialización a comparación de los hombres, aunque es aceptable (comparativamente con el hombre) la inclusión de la mujer en este sector.

\section{b) Caracterización en el mercado de trabajo en el que se desenvuelven.}

En el país según un estudio realizado por el Global Entrepreneurship Monitor (GEM), 2014)3 , señala que la actividad emprendedora en su mayoría es ejercida por el hombre, asumiendo como natural la forma tradicional y masculina en hacer negocios, es importante también mencionar que el hombre emprende más por oportunidad y la mujer por necesidad, como una última alternativa en generación de ingresos propios.

\section{Gráfico 2}

\section{Dependencia de la mujer en el mercado de trabajo}

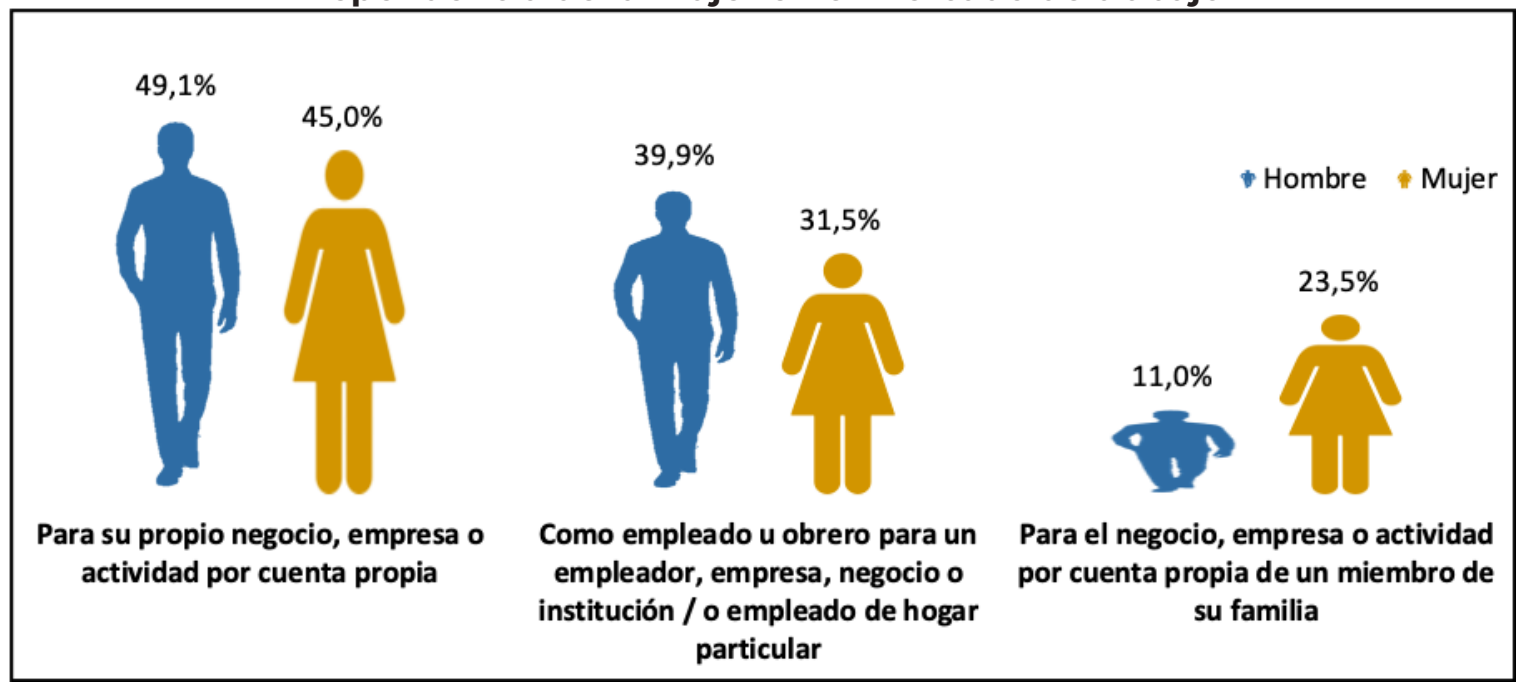

Fuente: Metadatos INE-ECE-3T2019/Elaboración: propia

Hay más mujeres dependientes de un negocio pequeño familiar (23,5\%), frente al (11\%) de los hombres, tenemos a más hombres ocupando cargos más estables a nivel incluso institucional (40\%) las mujeres con (32\%); en ambos sexos se puede observar que casi la mitad de la población en Bolivia se dedica a la actividad por cuenta propia.

2 Encuesta realizada por Agencia Española de Cooperación Internacional para el Desarrollo (AECID) Instituto Internacional para la Democracia y la Asistencia Electoral (IDEA Internacional), a través de la Coordinadora de la Mujer

3 Global Entrepreneurship Monitor (GEM), una investigación internacional que se realiza desde 1997 bajo la tutela del Babson College (Estados Unidos) y la London School of Business (Reino Unido), con el objetivo de medir el emprendimiento en diferentes países y observar en qué grado esta actividad afecta la tasa de crecimiento económico y la prosperidad de un país. El GEM busca, además, identificar los factores que promueven o inhiben la actividad emprendedora como un insumo para las políticas públicas. 


\section{Gráfico 3}

Formas en las que trabaja la mujer en el mercado de trabajo

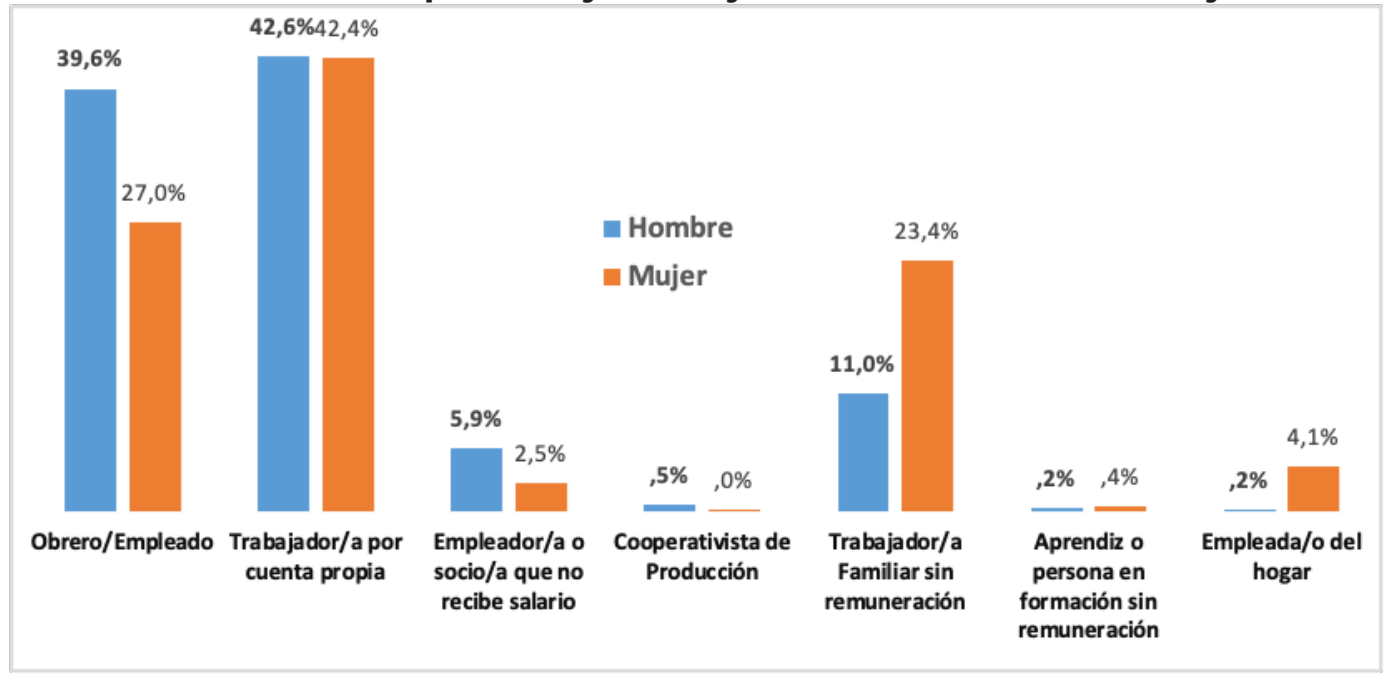

Fuente: Metadatos INE-ECE-3T2019/Elaboración: propia

Cerca del 25\% de las mujeres en Bolivia trabaja, pero no recibe ningún ingreso si a esto sumamos el sector de trabajadores del hogar, podemos señalar que casi el 30\% de las mujeres bolivianas realiza trabajos del hogar. El (40\%) de los hombres cuentan con trabajo estable frente al (27\%) de las mujeres, tenemos a más hombres que forman sociedades y son empleadores.

\section{c) Características de los negocios de las mujeres emprendedoras.}

Haciendo un análisis a los reportes brindados por FUNDEMPRESA 4 al finalizar la gestión 2019, podemos observar que Bolivia desde el año 2000 está compuesta con un promedio del 80\% de su base empresarial de empresas unipersonales, desde las características de los mismos una empresa unipersonal está compuesta por un solo socio por ende su inversión en capital suele ser pequeño, el tamaño de la empresa también es pequeño al igual que su producción, por estas características no demandan mucho trabajo como lo hacen las Sociedades de Responsabilidad Limitada que son empresas más estables pero sin duda aquellas que generan un mayor movimiento son las Sociedades Anónimas y las Colectivas, en términos porcentuales de participación en la base empresarial estas dos últimas ocupan índices insignificantes teniendo el 1,08\% de participación, las unipersonales en las gestiones 2013 y 2015 inclusive llegaron al 90\% de la base empresarial.

A ello señalamos que Bolivia cuenta con un trabajo informal preocupante, el INE no establece cuanto es el trabajo informal al menos no existe información oficial, a nivel del Ministerio de Planificación mencionaban el 2017 que existía un 70\% de mercado informal, Según la publicación del Fondo Monetario Internacional (FMI) el año 2015, en "Economías Sombrías del Mundo" cataloga a Bolivia como el país con mayor informalidad de mercado del mundo con $62.3 \%$ del Producto Interno Bruto (PIB), si analizamos los datos presentados hasta ahora y sumamos aquellos que se dedican en trabajos de cuenta propia, aquel trabajador sin remuneración y trabajadores del hogar coincidiríamos aproximadamente con los porcentajes señalados, el hecho es que en estas condiciones las mujeres emprendedoras realizan sus actividades.

$4 \quad$ FUNDEMPRESA es una fundación sin fines de lucro que opera el Registro de Comercio de Bolivia, brindando un servicio eficiente a los empresarios y al Estado, apoyando el desarrollo empresarial en Bolivia. el mismo certifica el nacimiento y legalidad de su empresa a través de la matrícula de comercio. 


\title{
144 Investigación \&egocios
}

\author{
Gráfico 4
}

Empresas formales e informales de las mujeres emprendedoras (aquellas que cuentan con NIT)

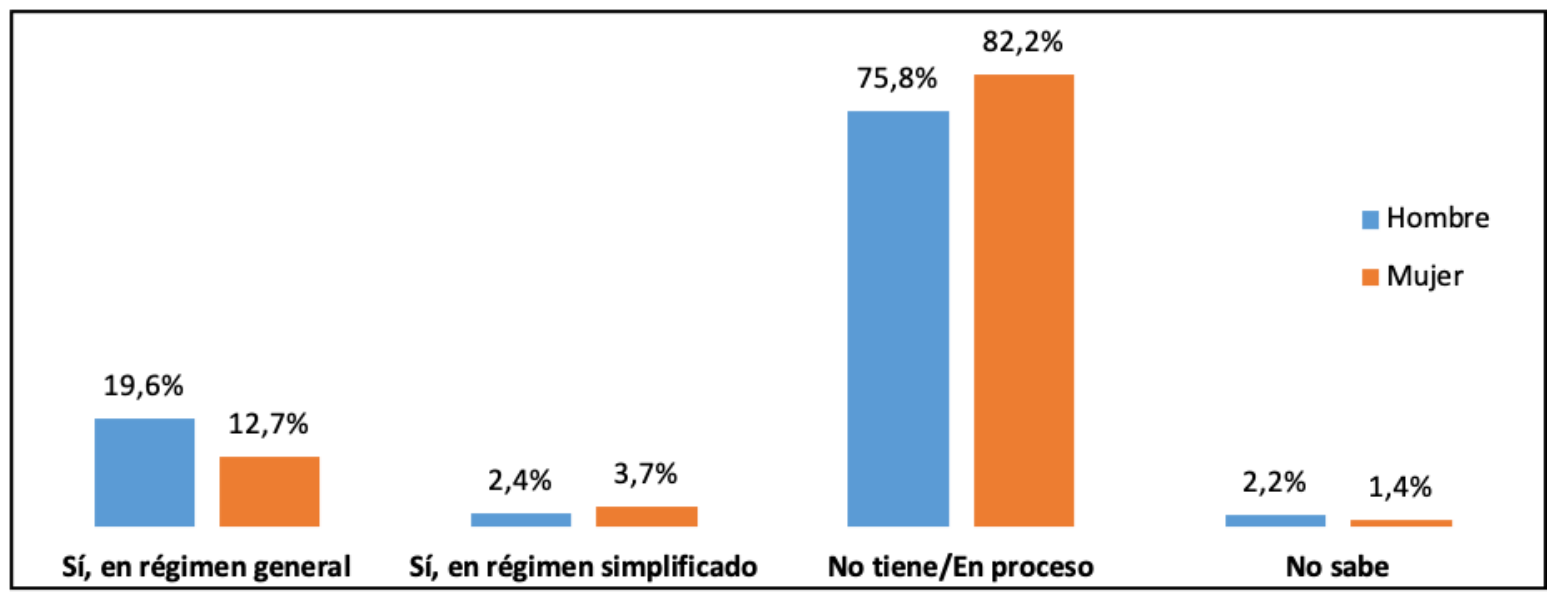

Fuente: Metadatos INE-ECE-3T2019/Elaboración: propia

Tenemos a más mujeres en el sector informal, el gráfico 4 muestra si la empresa cuenta con el Número de Identificación tributaria (NIT), claramente se confirma que Bolivia cuenta con muchas empresas en el sector informal; aunque existe la posibilidad que un porcentaje del mismo este en proceso de obtener su número de identificación.

\section{Gráfico 5}

\section{Lugar donde trabajan las mujeres de Bolivia (1)}

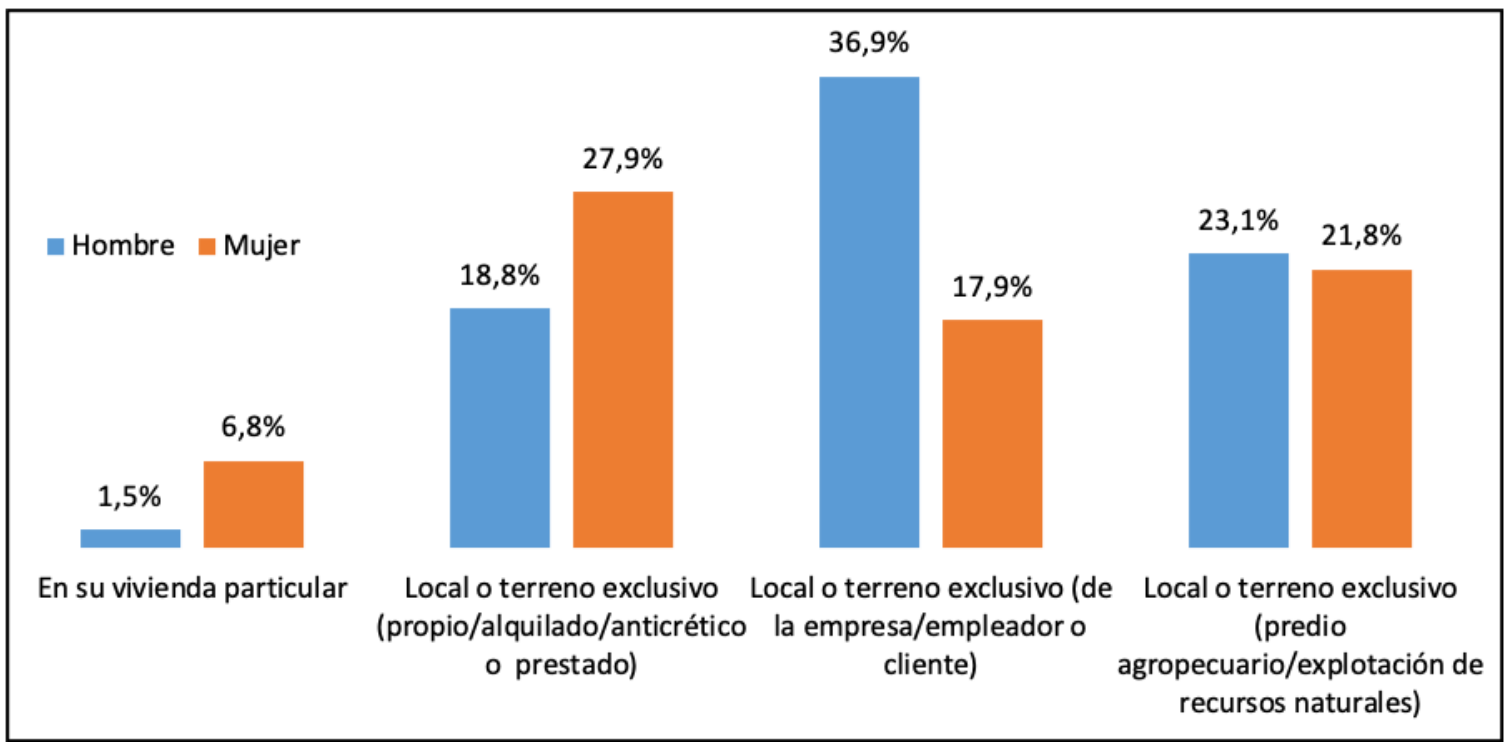

Fuente: Metadatos INE-ECE-3T2019/Elaboración: propia 


\section{Gráfico 6}

Lugar donde trabajan las mujeres de Bolivia (2)

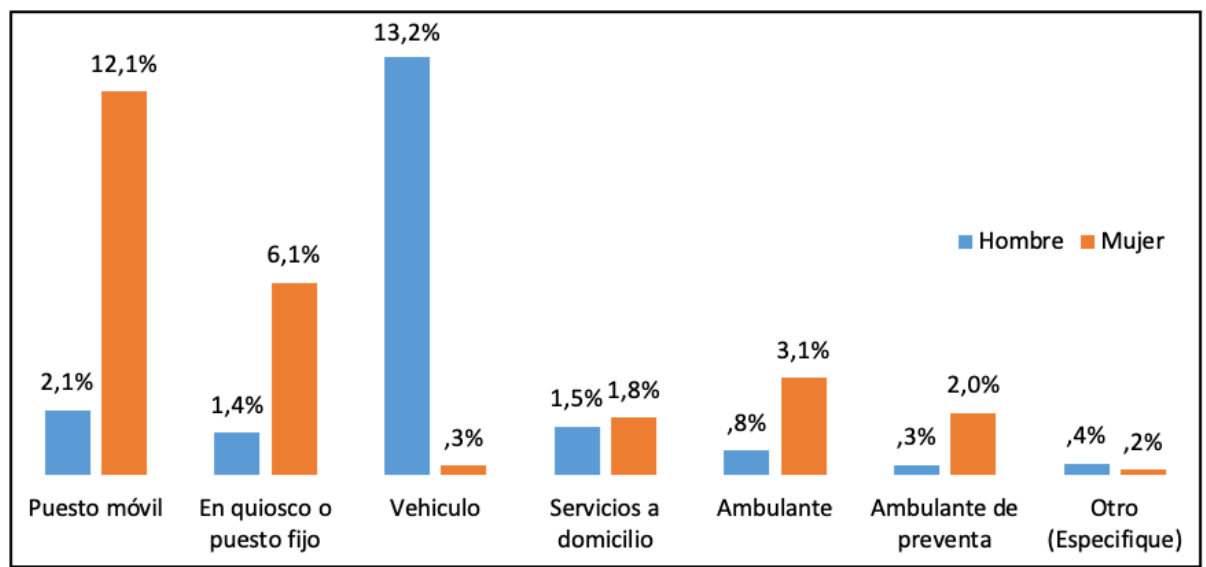

Fuente: Metadatos INE-ECE-3T2019/Elaboración: propia

El 37\% de los hombres trabajan en empresas o instituciones, pudiendo afirmar que cuentan con cierta estabilidad laboral, mientras el $28 \%$ de las mujeres trabajan en ambientes de terceros es decir existen más mujeres emprendedoras inclusivamente al interior de sus propias viviendas $(6,8 \%)$, hay más mujeres con puestos móviles $(12,1 \%)$ aquellas que trabajan en quioscos o puestos fijos (6,1\%), mujeres ambulantes (3,1\%) o aquellas que trabajan en preventas (2\%); mientras esto sucede con las mujeres los hombres están en sus vehículos trabajando (13,2\%), haciendo visible por lo descrito que la asignación de roles que explica el patriarcado por el actuar de la sociedad aún presenta brechas en el mercado de trabajo; haciendo un análisis general con los datos estadístico aproximadamente el $60 \%$ de las mujeres en edad de trabajar ocupan puestos o cuentan con emprendimientos, pequeños, con una mayor propensión al riesgo y sumar a ello que la mayoría se encuentra en un mercado informal, bajo estas características mencionadas los hombres están con cerca al $25 \%$.

Aproximadamente el 13\% de mujeres y hombres en edad de trabajar cuentan con un segundo trabajo, este segundo trabajado está caracterizado en la informalidad, algo importante de destacar es que, la mujer cuenta con mayor asistencia en el seguro de salud brindado por la fuente laboral con 42,9\% frente al 38,8\% de los hombres, sin embargo hay un buen porcentaje de mujeres $(57,1 \%)$ y hombres (61,2\%), que no cuentan con seguro de salud brindada por la fuente laboral.

\section{Gráfico $\mathbf{N}^{\circ} 7$}

\section{Aportes a la Agencia de Fondos de pensiones (AFP)}

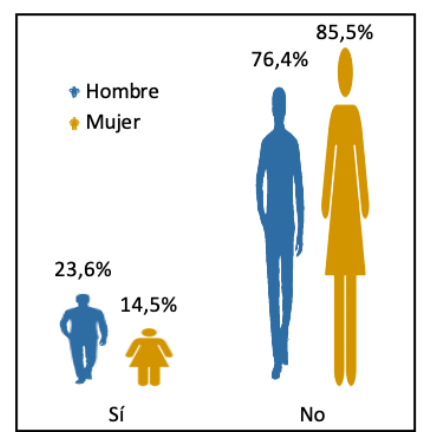

Fuente: Metadatos INE-ECE-3T2019/Elaboración: propia 


\section{Investigación Negocios}

Se evidencia que solo el $14,5 \%$ de las mujeres en edad de trabajar hacen el aporte a la AFP, más del 85\% no lo hace, el gráfico 7 señala que un buen margen de la población boliviana no aporta a la agencia de fondos.

Observamos las debilidades del sector emprendedor y empresarial, muchas instituciones a nivel Nacional como las Cámaras de Industria y Comercio, las Federaciones de Empresarios Privados atribuyen que este flote del mercado informal es efecto de los procesos y carga impositiva que deben soportar las empresas nuevas, políticas públicas que permitan una mayor inversión en la nación; a esto debemos mirar hacia el interior de las empresas y negocios de las mujeres emprendedoras sean que estén en el mercado formal o informal.

\section{Gráfico $\mathbf{N}^{\circ} 8$}

\begin{tabular}{|c|c|c|c|}
\hline \multirow[b]{2}{*}{ \& Mujer } & & $76,9 \%$ & \multirow[b]{4}{*}{$1,3 \%$} \\
\hline & & & \\
\hline \multirow{2}{*}{$12,8 \%$} & $8,9 \%$ & & \\
\hline & & & \\
\hline $\begin{array}{l}\text { Se llevan libros de } \\
\text { contabilidad o se } \\
\text { acude a los servicios } \\
\text { de un contador }\end{array}$ & $\begin{array}{l}\text { Sólo se utiliza un } \\
\text { cuaderno personal } \\
\text { para llevar las } \\
\text { cuentas }\end{array}$ & $\begin{array}{c}\text { No se lleva registro } \\
\text { contable }\end{array}$ & $\begin{array}{l}\text { No sabe / No } \\
\text { responde }\end{array}$ \\
\hline
\end{tabular}

Fuente: Metadatos INE-ECE-3T2019/Elaboración: propia

Las capacidades de gestión de una empresa están basadas en los principios de la administración, y una de ellas rige el proceso de administración y control, observamos que 77\% de las mujeres trabaja en negocios donde no se lleva los registros contables y como observamos en el gráfico 6 un gran porcentaje de las mujeres se encuentran en la informalidad, donde no realizan estos procesos administrativos, a esto podemos verificar que el 13\%, de las mujeres trabajan en empresas o son propietarias de la misma dentro del mercado formal, cumpliendo los requisitos que la normativa señala para la constitución y funcionamiento de una empresa. Aquellas mujeres que se dedican a una segundo oficio las características son las mismas, no llevan una gestión administrativa y cuando lo hacen es de manera empírica. 
Gráfico $\mathbf{N}^{\circ} 9$

Ingresos económicos de las mujeres

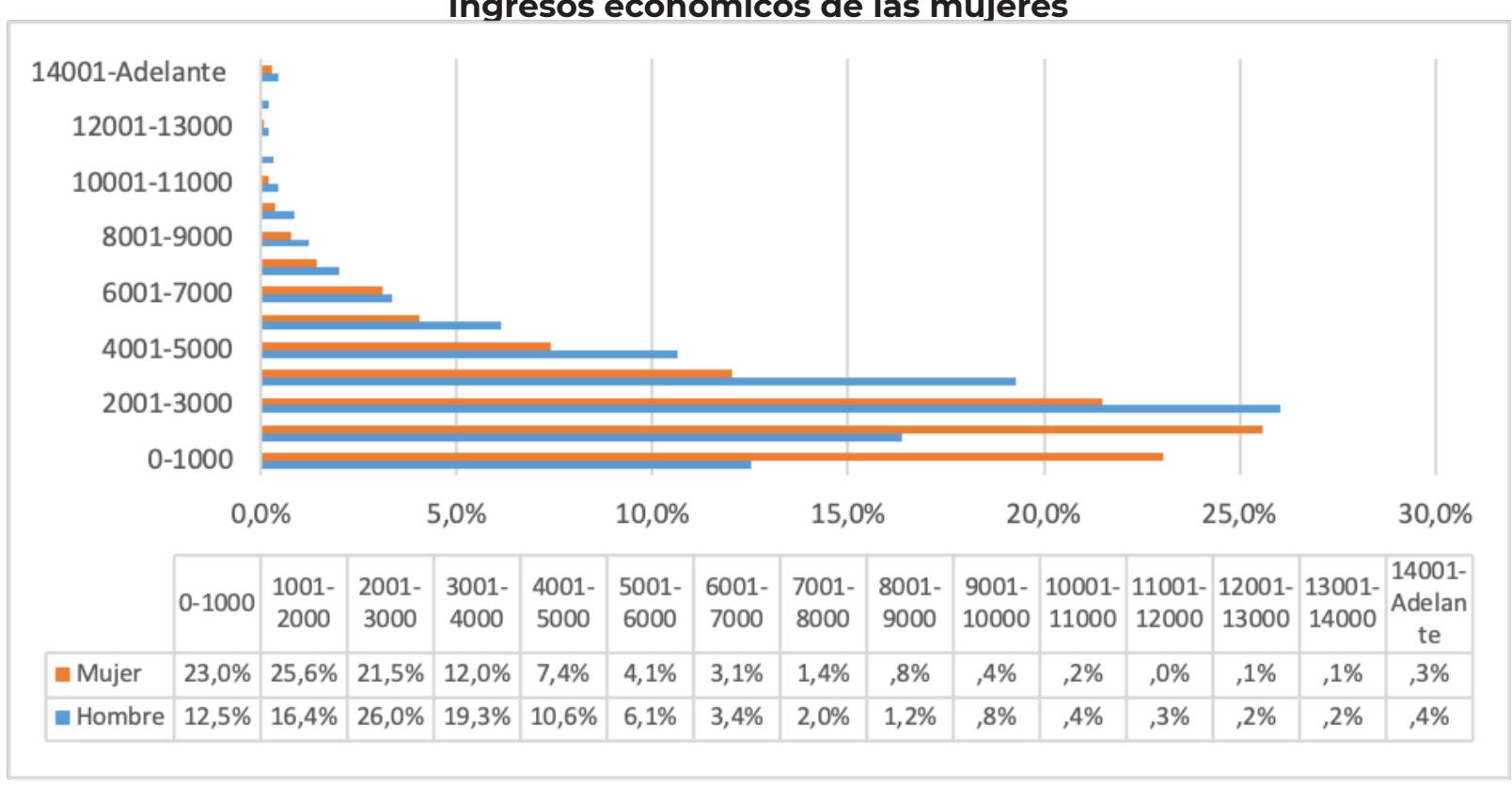

Fuente: Metadatos INE-ECE-3T2019/Elaboración: propia

En Bolivia según los datos mostrados, en promedio, el 60\% de la población femenina cuenta con ingresos laborales menos que el mínimo nacional, el $28 \%$ de las mujeres obtiene ingresos de Bs.3000 a 8000 , frente al $42 \%$ de los hombres son muy pocas las personas (hombres y mujeres) que obtienen ingresos mayores de Bs. 10000; los mayores ingresos laborales arriba de los Bs. 15000 recae en los hombres con una representación de $0.4 \%$ y las mujeres con 0.3\%.

Desde el punto de vista técnico de la estadística estos resultados se asemejan comparativamente a la Encuesta de Discriminación y Exclusión Social aplicada por la Coordinadora de la Mujer, (2014); donde, se mencionaba que el 13,30\% de la población femenina no contaba con ingresos, la mayor cantidad el $64 \%$ de la población femenina recibía ingresos menos de mínimo nacional y que apenas 0,2\% recibía ingresos mayores de Bs.10000.

El estudio del Panorama Social de América Latina, elaborada por la CEPAL, (2014); refleja la existencia de brechas en Bolivia sobre los ingresos entre hombres y mujeres, siendo el ingreso mensual en las mujeres menos en un 50\% a comparación de los hombres.

La principal fuente de ingresos en las familias de América Latina, son sueldos y salarios, que corresponden un 54\% del volumen total de sus ingresos, además que en la región (América Latina y el Caribe) una de cada dos mujeres en edad de trabajar tiene empleo o lo busca es decir es económicamente activa.

Tal como se manifestó en el análisis otra fuente de obtención de ingresos es en el ámbito empresarial y Financiero y las ganancias del trabajo de forma propia e independiente, en donde las mujeres tienen una mayor participación sin embargo en un mercado informal, haciendo que los niveles de ingresos sean más propensos a desaparecer (CEPAL, 2016).

\section{Reinvención de la mujer emprendedora-una reflexión desde la experiencia del autor.}

No existe un país que no se vea afectado por la pandemia producto de la COVID-19, pasaron 7 meses desde que un mercado en China cerró sus instalaciones producto de los contagios masivos traduciéndose en el primer efecto económico, ahora se habla de un shock en el mercado 


\section{Investigación Negocios}

de trabajo traducido en millones de despidos, economías llamadas potencias afectadas micro y macroeconómicamente; los datos negativos las observamos diariamente en diversas instancias.

La crisis sanitaria y los primeros golpes de la pandemia se dejaron sentir en Bolivia en las primeras semanas del mes de marzo, al finalizar el 2019 la economía boliviana terminaba con indicadores económicos decrecidos en comparación del último trimestre del 2018 y 2017, una parte de ellos fue fruto de los acontecimientos políticos y las convulsiones sociales que terminó con una presidencia transitoria y por demás criticada, el 2020 prometía una recuperación especialmente por el lado de la oferta agregada, sin embargo la presencia de la pandemia golpeó principalmente a las variables de Consumo, Inversión, Exportaciones e Importaciones, afectando en sí a la demanda agregada de Bolivia

Es importante mencionar que los departamentos de La Paz y Santa Cruz en más de 14 años dentro su estructura productiva presenta una mayor diversificación en relación al sistema nacional, departamentos como Chuquisaca, Pando, Potosí y Oruro, cuentan con una estructura menos diversificada, donde el efecto dinamizador de la inversión privada es muy pobre, a esto sumar que la economía boliviana cuenta con altos índices de empleo informal de hecho el Banco Interamericano de Desarrollo (BID), mencionó que la crisis del desempleo provocado por el COVID-19 afectará más a países con una alta tasa de informalidad, y que Bolivia tiene en riesgo aproximadamente 5.2 millones de empleos, de esta cantidad 4.2 millones correspondientes al mercado informal.

En estas condiciones se habla de la reinvención que debe tener el sector empresarial, adecuadas a la utilización de medios tecnológicos, se habla de adaptación, de las nuevas formas para llegar al cliente, de contar con canales de comunicación y de comercialización con una alta injerencia en la utilización de redes sociales, software, aplicaciones y otros; es cierto, la no utilización de medios tecnológicos, y el no poder adaptarse, traerá consigo la muerte de muchas empresas, y en Bolivia habrá dos caminos como efectos de la pandemia, se incrementará el mercado informal que es lo más probable o si se busca las políticas necesarias para el sector privado se incrementará las empresas unipersonales; es aquí donde la condición de la mujer tiende a ser desfavorable, es donde radica el análisis crítico de esta investigación.

La CEPAL en el documento "la autonomía de las mujeres en escenarios cambiantes" publicada al finalizar el 2019 expresa algunas tendencias en el ámbito tecnológico como alternativas y formas en la reinvención de las mujeres en tiempos cambiantes.

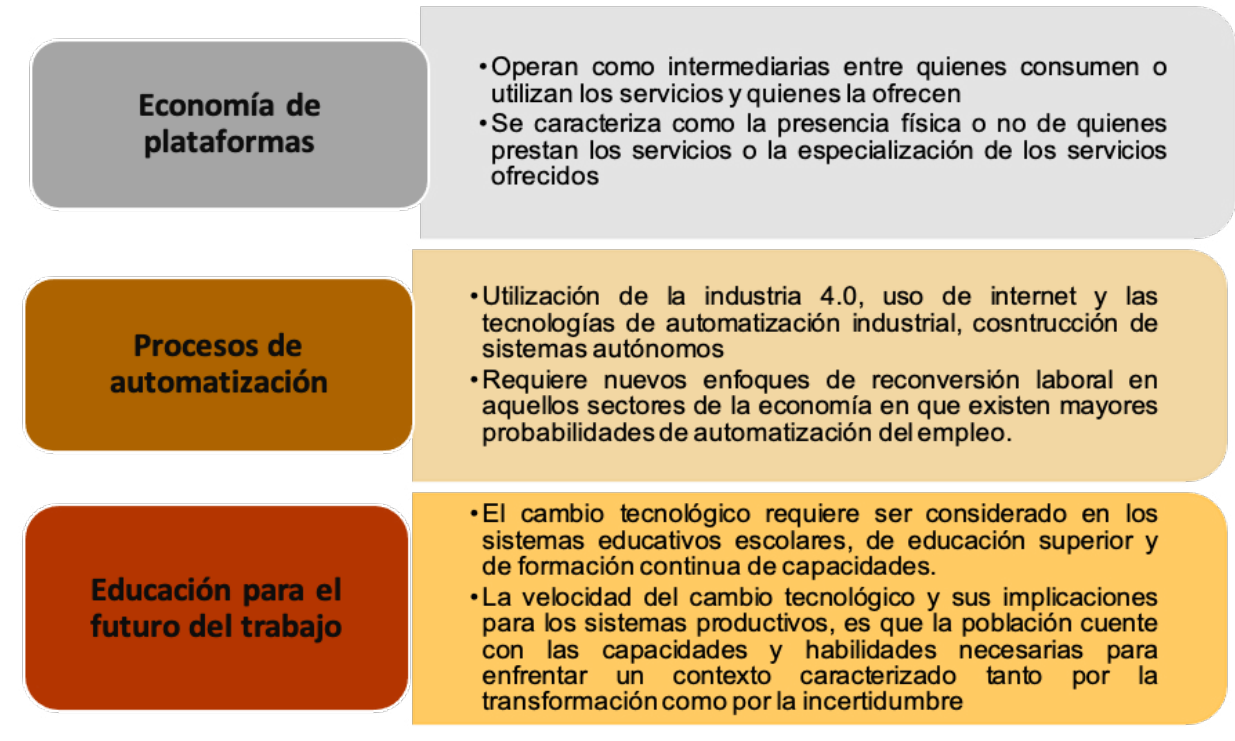

Fuente: CEPAL, 2019/Elaboración: Propia 
Las nuevas formas de trabajo avanzan a medida de la tecnología y la innovación es menester señalar que las mujeres se verán más afectadas en esta readaptación si bien el alcance en la educación en Bolivia ha mejorado hacia la inclusión de la mujer no es suficiente, porque la mayoría solo terminó la secundaria, se habla de plataformas, de tecnologías relacionadas a la eficiencia de las cadenas productivas, las nuevas formas de aplicación en redes sociales, blogs, la readaptación del marketing en función a la plaza, producto, precio, etc.; sin embargo nuestra realidad boliviana pone en manifiesto a una cantidad de mujeres dedicadas al mercado informal, mujeres ambulantes, con puestos o quioscos, aquellas que no cuentan con un puesto fijo, aquellas que cuentan con dos oficios en las mismas características con emprendimientos precarios, se habla de "reinvención"; cómo puede una mujer acceder a todas estas recomendaciones que la teoría señala si al margen de dedicar tiempo a esta adaptación tiene que dedicarse al trabajo del hogar, donde no son reconocidas, menos contabilizadas económicamente.

Se generan espacios de capacitación que pueden ser pagados o no, pero la mujer, se ve con más roles al interior de su hogar, dedicadas al emprendimiento, a las labores al interior de su hogar, al cuidado y atención de los hijos, ahora, tratar de adecuarse y capacitarse para que su negocio no cierre, o idearse nuevas formas para llegar a ofertar su producto o servicio.

Cómo puede readecuarse una mujer emprendedora a las plataformas digitales, por ejemplo con el teletrabajo si un gran porcentaje Bolivia de mujeres empresarias no cuentan con las habilidades y conocimientos suficientes para la optimización de sus cadenas productivas (tecnología 4.0), además la estructura de su negocio no encaja con estas nuevas formas, porque tienen una gestión de empresas muy tradicional, según los datos analizados cerca del $17 \%$ de las mujeres emprendedoras o en edad de trabajar cuenta con una empresa más estable, tampoco es sinónimo de sostenibilidad pos pandemia, según el GEM, las empresas en Bolivia cuentan con una alta probabilidad de quiebra en los primeros 3 años, y vista desde el punto de la experiencia este comportamiento efecti- vamente se cumple, puesto que en promedio los flujos proyectados en los proyectos de inversión muestran recuperación a partir del tercer año, "hablamos de recuperación de la inversión", esto quiere decir que a partir del cuarto y quinto año una empresa nueva recién obtiene utilidades netas; sin duda estos parámetros en una empresa se modificaran y los periodos de recuperación de inversión serán más largos.

Cuando se habla de la educación para el futuro del trabajo, implica los conocimientos y el grado de especialización con las que deben contar las mujeres emprendedoras para la gestión de su emprendimiento y más si se encuentra en el mercado formal; en los primeros tres meses de confinamiento en Bolivia, los empresarios a nivel nacional solicitaron apoyo mediante créditos para cubrir los salarios de sus trabajadores, por esta situación podemos señalar que a nivel nacional la mayoría de las empresas no manejan el capital de trabajo, que por lo menos debería cubrir los primeros meses en el funcionamiento de la empresa dependiendo de la rotación de stock que tenga, por lo contrario los créditos deberían de destinarse para la optimización de las empresas y la generación de una mayor inversión, más allá de ello, tras el trabajo que se tuvo en anteriores investigaciones, se pudo determinar que las mujeres emprendedoras cuentan en su mayoría con conocimientos empíricos de gestión empresarial y el manejo de las capacidades de su negocio ya sean administrativa, operativa y comercial; el problema es que, cuando el negocio es pequeño no requiere mayores capacidades, pero, a medida que el negocio crece, necesitan de otras habilidades incluso organizativas, y si este negocio pasa a formar parte de un tipo societario tienen otras necesidades al interior de sus empresas, como asesorías legales, ambientales, de marketing, de gestión etc.

Debemos entender ahora que las mujeres emprendedoras tienen un camino difícil de recorrer con sus negocios, según el reporte del INE en la segunda quincena del mes de julio, mencionó que el desempleo se incrementó un poco más del 8\%, un índice similar al que se presentó en los periodos críticos de nuestra historia nacional, donde los niveles de pobreza y pobreza extrema eran desesperantes, posterior a ello planteada la Agenda Pa- 


\section{Investigación \& Negocios}

triótica en el Estado Plurinacional de Bolivia, se mencionaba que la pobreza tenía rostro de mujer; la reinvención de las mujeres bolivianas al menos de la mayoría que cuentan con emprendimientos precarios, emprendimientos creados por la necesidad, va a consistir en tratar de sostenerse en una economía golpeada, en tratar de acomodarse en otros rubros, en síntesis, la "reinvención" aquella que para algunos representará la utilización de medios tecnológicos y plataformas, para aquellas que carecen de capacidades significará solamente una adaptabilidad precaria.

Pero la carencia de capacidades está en el sentido de la formación educativa, la crisis está llevando a que los procesos de formación tengan un salto cualitativo, la adaptabilidad o reinvención en las mujeres está haciendo incluso que el trabajo laboral en ellas no esté amparado por la ley general del trabajo, porque la carga adicional que tiene la mujer en la reinvención o la adaptabilidad no está siendo reconocida y esto pasa en diferentes escenarios. De este modo, no se resuelven problemáticas tradicionales que están asociadas con el trabajo desde el hogar, como el aislamiento, las condiciones precarizadas de empleo, las dificultades para la organización y sindicalización, y la falta de regulación y fiscalización laboral (Hunt y Samman, 2019).

La autonomía económica de las mujeres en Bolivia se encuentra en una fase crítica, infiriendo; si el 60\% de las mujeres cuentan con ingresos laborales por debajo del mínimo nacional (Bs.2.122), considerando que la jornada laboral en Bolivia es en promedio Bs.70 y que el confinamiento rígido fue más de tres meses, la jornada se ve afectada cerca del 30\%, este porcentaje es menos de la mitad porque el sector de mercado informal se dio modos para seguir trabajando, por eso más que hablar de reinvención se habla de adaptabilidad bajo escenarios cambiantes.

Se habla mucho de políticas, de hecho Bolivia trabajó mucho el tema de poder proteger a la mujer desde los sistemas políticos, salud y educación principalmente, pero no existe una política que vaya directamente al fortalecimiento de los emprendimientos de la mujer bajo la consigna de consolidar su autonomía económica, no se ha mejorado el mercado informal, se cuenta con muchas empresas unipersonal, no es malo, pero, diversas instituciones trabajan en la dotación de capital semilla, brindado capacitaciones, asesoramientos, de una manera aislada y dispersa, creando únicamente pequeños negocios que luego al salir al mercado suelen ser absorbidos o permanecen como emprendimientos precarios, son muy pocas mujeres que trabajan en una fuente laboral estable y sólida, se ha demostrado con los datos, es decir que estos programas de ayuda no están teniendo los efectos necesarios para alcanzar una autonomía de la mujer, estos programas de ayuda hacia la mujer con tipologías que llegan a ser interesantes como las incubadora de empresas de emprendimientos y otros, solo se han traducido en más mercado informal y en más empresas unipersonales, urge un cambio de pensamiento y estructura de fortalecer a estas empresas unipersonales para que se constituyan en una sociedad más grande, y que aquellos que están en un mercado informal pasen a ser la nueva estructura de las unipersonales.

Bajo la práctica investigativa que se ha desarrollado en otros temas como sujeto investigador, se menciona que la economía de Bolivia tendrá una leve mejora al finalizar el tercer trimestre, dependerá de las acciones que se tomen, en el corto plazo urge medidas que dinamicen la demanda agregada y en el mediano plazo urge de políticas que dinamicen la oferta agregada, la inversión y la capacidad productiva de Bolivia; el gasto público en el corto y mediano plazo es inminente y los niveles de endeudamiento pudiese alcanzar niveles vistos en los periodos llamados neoliberales.

Indiscutiblemente la autonomía de la mujer en estos periodos del shock COVID-19 en Bolivia serán afectados, no se garantizará su autonomía economía, porque incluso las medidas económicas propuestas por el gobierno transitorio están destinados a cubrir necesidades de otro tipo de sectores, sin embargo como mencionaba Remollins, una emprendedora es aquella que puede levantarse desde una situación económica precaria, ellas siguen siendo emprendedoras, líderes, capaces de adaptarse, pero, sin autonomía económica. 


\section{Bibliografía.}

- Anker, R., (1996) "La Segregación profesional entre hombres y mujeres Repaso de las Teorías". En Revista Internacional del Trabajo, Vol. 116, núm.3, pp. 343-370.

- Bravo, R., (2012)"Avances y Desafíos en torno a la Autonomía Económica de las Mujeres". Ponencia presentada en el Panel "Avances y Desafíos en torno a la Autonomía económica de las Mujeres del Seminario Género en el Poder". En Chile de Michelle Bachelet. Organizado por el Observatorio Género y Equidad

- Calderón, C., (2017) Construyendo el perfil de la mujer emprendedora en Sucre: un avance para su autonomía económica, Bolivia, Universidad San Francisco Xavier de Chuquisaca, tesis de maestría.

- Carrasco I., (2008) "El Emprendedor Schumpeteriano y el contexto Social". Universidad de Castilla-La Mancha. ICE, número 845, pp.121-134

- Centro de Investigaciones Sociales (CIS) y Ciudadanía, Comunidad de Estudios. (2017). Encuesta Mundial de Valores en Bolivia 2017 (Primera edición ed.). (C. D. Sánchez, Ed.) La Paz, Bolivia: Vicepresidencia del Estado Plurinacional de Bolivia.

- CEPAL, (2016) "Autonomía de las Mujeres e igualdad en la Agenda de desarrollo Sostenible". Santiago

- Cobo, R. (1995) "Género" en Celia Amorós (Dir.) 10 palabras claves sobre mujer. Madrid: Editorial Verbo Divino.

- Comisión Económica para América Latina y el Caribe (2019) La autonomía de las mujeres en escenarios económicos cambiantes (LC/CRM.14/3), Santiago.

- Correa, E. (2014) "Sociedad patriarcal, las luchas por la equidad de género y el posneoliberalismo" en Alba Carosio (Dir.) Feminismos Para Un Cambio Civilizatorio. Fundación Centro de Estudios Latinoamericanos Rómulo Gallegos. Caracas: Fundación Imprenta de la Cultura.

- Espinar, E., (s/f) Violencia de Género y Procesos de Empobrecimiento. Tesis Doctoral. Departamento de Sociología Universidad de Alicante.

- Ferran, P., Guillem, P. y T. Albert, (2015) Es- cuela de Emprendedores. España: Lexus.

- Fontenla M., (2008) "Qué es Patriarcado - Claves del feminismo" en Diccionario de Estudios de Género y Feminismos. Argentina: Editorial BIBLOS

- Hartman, H., (1987) "Un Matrimonio mal avenido: Hacia una unión más progresiva entre Marxismo y Feminismo" en Cuadernos del Sur, núm. 6, Buenos Aires, Marzo-Mayo.

- Heller, L. (2010) Mujeres emprendedoras en América Latina y el Caribe: realidades, obstáculos y desafíos, Mujeres y Desarrollo. Serie mujer y desarrollo 93. Chile: División de asuntos de género.

- Hernández, J. (2008) "Género y educación para la paz: tejiendo utopías posibles" en revista Venezolana de Estudios de la Mujer. Vol. 13, p.p 79-98. Caracas: Universidad Central de Venezuela. Centros de estudios de la mujer.

- Herrera C., y L. Montoya, (2013) “El emprendedor: una aproximación a su definición y caracterización". Universidad Nacional de Colombia, núm. 7, pp. 7-30

- Hunt, A. y E. Samman (2019), "Gender and the gig economy", Working Paper, $\mathrm{N}^{\circ}$ 546, Londres, Overseas Development Institute (ODI), enero.

- Medina, L., y Friedrich, S. (2018). Shadow Economies Around the World: What Did We Learn Over the Last 20 Years? International Monetary Fund.

- Querejazu, V. et al., (2015) Global Entrepreneurship Monitor - Reporte Nacional Bolivia 2014. Universidad Católica Boliviana "San Pablo". La Paz: Artes Gráficas Sagitario SRL.

- Ramos P. M. Dolores, (1995) "Historia social: un espacio de encuentro entre género y clas". En fundación Dialnet, Número 27, pp. 85-102.

- Remolins E., (2015) Aventura de Emprendedores. España: Lexus.

- ONU y Ministerio de Autonomías, (2015) "Las Mujeres escribiendo nuestra Autonomía". Segunda Edición. La Paz

- Osorio F., (2010) "La estrategia y el emprendedor: diversas perspectivas para el análisis". Universidad del Valle Colombia, núm. 43, pp. 65-80 